\title{
The Impact of COVID-19 on Preclerkship Teaching
}

\author{
Hina Mohsin ${ }^{1,2}$, Dina Saba ${ }^{2}$, Fauzia Nausheen ${ }^{1,3}$, Mohsin Yakub ${ }^{1 *}$ \\ ${ }^{1}$ California University of Science and Medicine, USA \\ ${ }^{2}$ University of California Riverside, USA \\ ${ }^{3}$ California Health Science University-COM, USA
}

*Corresponding author: Mohsin Yakub, Assistant Dean of Admissions, Associate Professor of Medical, Education: Physiology and Nutrition, California University of Science and Medicine, 217 E. Club Center Drive, Suite A. San Bernardino, CA 92408, USA.

To Cite This Article: Mohsin Yakub. The Impact of COVID-19 on Preclerkship Teaching. 2020 - 9(2). AJBSR.MS.ID.001380. DOI: 10.34297/ AJBSR.2020.09.001380.

Received: 海 June 17, 2020; Published: 笽 June 22, 2020

\section{Introduction}

Almost every system is impacted due to COVID-19 pandemic. The clinical aspect of the medical education system is also paying an unavoidable price. Here is an insight into the rapid transition in pre clerkship teaching from onsite to the online environment. This sudden shift in teaching away from the classroom was not a choicerather a need, for which the learners and educators were not well prepared. At the beginning of this transition some believed that this impromptu move to virtual learning, without planning, would result in chaos. However, few see this as an opportunity to introduce a hybrid curriculum in pre-clerkship years, with extra benefits for the learners which include but not limited to content control, sequencing, pace, time management, and the control to tailor them to meet their personal learning objectives [1]. Herein, we describe three teaching pedagogies (small group settings) that we continue during the pandemic with suitable alterations. Also, we describe the student and faculty insight into the challenges and how the challenges related to some of the benefits of online medical education. Knowledge of these challenges can be applied in the design, structure and moderation of online discussions to minimize the inconveniences and limitations posed by the use of these technologies.

a. Clinical Skills: During the pandemic, this class has been switched to online meetings via Zoom or Microsoft team. Each group is comprised of 4-5students, facilitator, and a standardized patient (SP).The sessions consist of a history taking, creating differential diagnosis, management, plan, and assessment. The limitation was on the physical examination which is not possible virtually. To compensate for that, we played an examination video and discussed the maneuvers with the group and SPs. During the virtual OSCE, students took history from SP, which was graded by facilita tors on exam soft app. Moreover, for the physical examination, we conducted a survey comprised of open answer questions which was quiz based on physical examination videos.

b. Problem Based Learning: This class was switched to online small group meetings. The premise stayedthe same with two minor changes: - a)no designated note-taker during class and b) the case was presented via PowerPoint instead of Word document.

c. Doctoring: In doctoring sessions, students become familiar with ethical issues, professionalisms, public health, and global health. In the session, one or two students interview the receive feedback from both facilitators and the SP.

Transitioning to online was the same except the standardized patient encounter was simulated by a telemedicine video call. The students had inconsistent experiences completing e-learning during the courses. Despites of many satisfied students, some still see in person Clinical Skills, PBL, and Doctoring as superior because of the interactive learning environment and communication without any barriers [2]. Students perceive that in person small groups are more engaging and results in pertinent information from participants being easier to remember due to the environment. There are worries among students that missing Clinical Skills will not prepare them to enter rotations in their third year.

Various methods of communication are available during online teaching like a chat box and audio tools that creates chaos when students accidentally speak over each other to ask questions. Additional challenges to e-learning are equipment availability and internet connectivity. We foresee higher expectations for students about concerning with regard to technical competency and the ability to adapt. Hybrid learning will likely become commonplace with small groups in person and lectures online. 
The transition from the face to face teaching for Educators has required a great deal of preparedness and instructional strategies. Some of these challenges are related to establishing momentum in the discussion, digressions, and a lack of voice and facial expressions [3]. Other challenges include low rates of interaction and dialogue, in monitoring individual students' behavior and responses [2]. The increased use of email struggles with establishing boundaries between work and home, and lack of daycare support for kids are some additional challenges. Although there were challenges, we also witness that students had more motivation to participate in their own pace by self-directed learning and critical thinking and achieving higher levels of abstract cognitive processes $[4,5]$.

The pandemic appears to be the inflection point that is imposing disruption in how we teach medicine. Change and transformation are certainly coming. We must begin to think creatively about teaching, if the standard in-person preclinical course is not an option, and creating new opportunities for teaching, learning and delivering health care.
For educators, they require knowledge, training and comfort in the use of technology and building a community of learners with facilitating discussion and assessment. Overall, the current situation represents an enduring transformation in medicine that will contribute to the development of medical education in establishing of active curricular innovations and transformation.

\section{Reference}

1. Ruitz APA, Jorge G, Mintzer MD, Michael J, Rosanne M (2006) The Impact of E-Learning in Medical Education. Academic Medicine 81(3): 207-212.

2. Gudizal C (2002) Explaining the lack of dialogue in computer-supported collaborative learning. Paper presented at the Computer Supported Collaborative Learning Conference, CSCL.

3. Tiene D (2000) Online Discussions: A Survey of Advantages and Disadvantages Compared to Face-to-Face Discussions. Journal of Educational Multimedia and Hypermedia 9(4): 369-382.

4. Morgan M (2000) Getting Beyond the Chat: Encouraging and Managing Online Discussions.

5. Aviv R, Erlich Z, Ravid G, Geva A (2019) Network analysis of knowledge construction in asynchronous learning networks. Online learning $7(3)$. 